\title{
Adaptive Soundness of Static Equivalence ${ }^{\star}$
}

\author{
Steve Kremer and Laurent Mazaré \\ LSV, ENS Cachan \& CNRS \& INRIA Futurs \\ $\{$ kremer, mazare\}@lsv.ens-cachan.fr
}

\begin{abstract}
We define a framework to reason about implementations of equational theories in the presence of an adaptive adversary. We particularly focus on soundess of static equivalence. We illustrate our framework on several equational theories: symmetric encryption, XOR, modular exponentiation and also joint theories of encryption and modular exponentiation. This last example relies on a combination result for reusing proofs for the separate theories. Finally, we define a model for symbolic analysis of dynamic group key exchange protocols, and show its computational soundness.
\end{abstract}

\section{Introduction}

It is well-known that even simple security protocols are extremely error-prone. This is mainly due to the fact that they are executed in a hostile environment, e.g., the Internet. The need for rigorous proofs was recognized very early and two distinct, competing approaches have been developed. The symbolic approach considers an abstract model, where messages and cryptographic primitives are modeled by a term algebra. The adversary manipulates terms according to a pre-defined set of rules, typically an inference system. The computational approach considers a more detailed execution model. Protocol messages are modeled as bitstrings and cryptographic primitives are polynomialtime algorithms. The adversary is an arbitrary probabilistic polynomial-time Turing machine. Security of a protocol is measured as the adversary's success probability.

Proofs in the symbolic model can be (partially) automated, but it is not clear whether this abstract model captures all possible attacks. Proofs in the computational model provide stronger security guarantees but are generally harder and difficult to automate. A recent trend tries to get the best of both worlds: an abstract model with strong computational guarantees. In their seminal paper, Abadi and Rogaway [4] have shown a first such soundness result for symmetric encryption in the presence of a passive attacker.

Recently, Baudet et al. [9] presented a general framework for reasoning about sound implementations of equational theories. Instead of a fixed set of cryptographic primitives, they allow a specification by the means of an equational theory. The formal indistinguishability relation they consider is static equivalence, a well-established security notion coming from cryptographic pi calculi [3] whose verification can often be automated [2 10]. Studying soundness of equational theories is motivated by the numerous recent works on extending the classical Dolev-Yao result with equations which are intended to capture algebraic properties of cryptographic primitives (see [17] for a

\footnotetext{
* Work partly supported by ARA SSIA Formacrypt and ARTIST2 Network of Excellence.
} 
survey ). Showing a soundness result for an equational theory proves that indeed "enough" equations have been considered in the symbolic model.

In this paper we consider the question of soundness of static equivalence in the presence of an adaptive adversary, rather than a purely passive one. This extends the work by Baudet et al. in a similar way as the work of Micciancio and Panjwani [24] extended the work of Abadi and Rogaway [4]. An adaptive adversary is allowed to choose the messages whose implementation he will be given. The choice of the messages can hence depend on previously observed distributions. We illustrate the usefullness of such a model on dynamic group key exchange protocols.

More precisely the contributions of our paper are as follows. We define the notion of adaptive soundness of static equivalence in a general framework. The definition is parameterized by the equational theory and the concrete algebra implementing the symbolic model. Our notion is strictly stronger than the purely passive soundness from [9]. We also develop a combination based proof technique: it allows us to reuse soundness results of two disjoint abstract signatures and conclude soundness of the joint signature. While the conditions under which such a combination works are of course restrictive they nevertheless match cases of practical interest. We give adaptive soundness results for several theories: symmetric encryption provided that the encryption scheme respects a length-conceiling IND-CPA security notion (this is similar to the main result in [24]), exclusive or (XOR), modular exponentiation in an Abelian group provided that the Decisional Diffie-Hellman (DDH) assumption is verified. Finally, we use our combination technique to derive adaptive soundness for the joint theory of encryption and modular exponentiation. We believe these are the first adaptive soundness results for modular exponentiation. Their importance is motivated by real-life protocols such as SSL/TLS that rely on Diffie-Hellman key exchange and thus use modular exponentiation.

To illustrate the usefulness of adaptive adversaries we define a symbolic model for dynamic group key exchange (DKE) protocols. A DKE protocol is a suite of protocols which allows three actions: exchange of an initial key between a group of users, joining and leaving the group. A typical example of DKE is the AKE1 protocol [12]. In our symbolic model we assume static corruption, as it was the case in [24], and allow the adversary to schedule these subprotocol and decide which users initially exchange the key, join, respectively leave the group. We use our adaptive soundness result to show that this symbolic model is sound with respect to a corresponding computational model.

Related work. As discussed above this paper generalizes both work by Baudet et al. [9] and Micciancio and Panjwani [24]. Abadi et al. [1] also use the framework of [9] to show soundness of an equational theory useful for reasoning about offline guessing attacks modeled in terms of static equivalence. In [8], Bana et al. argue that the notion of static equivalence is too coarse and not sound for many interesting equational theories. As an example they show that the DDH assumption is not sufficient to imply soundness of static equivalence. They introduce a general notion of formal indistinguishability relation. In this paper we prefer to stick to static equivalence which has the advantage of being a well-established, tool-supported equivalence relation. We address the problems highlighted in [8] by proving soundness for a restricted set of well-formed frames (in the same vein Abadi and Rogaway used restrictions to forbid key cycles). Regarding the theory of XOR, Backes and Pfitzmann [6] have shown an impossibility result in 
the reactive simulatability framework with active attackers and a soundness result for passive attackers. Note that we use the same model as [9] and restrict ourselves to the XOR of pure random values and not arbitrary payloads. While this is a restriction, it may nevertheless be useful for computing keys as the XOR of two random values when combining XOR and encryption. Concerning modular exponentiation, a soundness result in the style of Abadi and Rogaway [4] has recently been shown in [13]. However using the same proof technique for the adaptive case seems difficult as the security model of [13] is tightly linked to the passive adversary model: challenges cannot be chosen adaptively by the adversary.

There have also been numerous works considering an active adversary using approaches. Without being exhaustive this work includes reactive simulatability providing universally composable results in [7]15], soundness results (but not universal composability) for an automated tool are presented in [18], cryptographically sound type systems [23], a Protocol Composition Logic in [19] and an automatic tool that aims at directly generating cryptographic proofs via sequences of games in [11]. However these works stick to classical cryptographic primitives: digital signatures, symmetric and asymmetric encryption. We are not aware of any general results for equational theories in the active case. Considering an active adversary is technically more involved although incomparable to an adaptive adversary. The case of a both active and adaptive adversary is a challenging problem and a topic of active research.

Because of lack of space, proofs are omitted. They are available in [22].

\section{Abstract and Computational Algebras}

We introduce our model, which is the same up to some minor changes as in [9].

\subsection{Abstract Algebras}

Our abstract models_-called abstract algebras — consist of term algebras defined over a many-sorted first-order signature and equipped with equational theories.

Specifically, a signature $(\mathcal{S}, \mathcal{F})$ is made of a set of sorts $\mathcal{S}=\left\{s, s_{1} \ldots\right\}$ and a set of symbols $\mathcal{F}=\left\{f, f_{1} \ldots\right\}$ together with arities of the form $\operatorname{ar}(f)=s_{1} \times \ldots \times s_{k} \rightarrow s$, $k \geq 0$. Symbols that take $k=0$ arguments are called constants; their arity is simply written $s$. We fix a set of names $\mathcal{N}=\{a, b \ldots\}$ and a set of variables $\mathcal{X}=\{x, y \ldots\}$. Names and variables are given with sorts and an infinite number of names and variables are available for each sort. The set of terms of sort $s$ is defined inductively by

$$
\begin{array}{ll}
t::= & \text { term of sort } s \\
x & \text { variable } x \text { of sort s } \\
a & \text { name } a \text { of sort s } \\
f\left(t_{1}, \ldots, t_{k}\right) & \text { application of symbol } f \in \mathcal{F}
\end{array}
$$

where for the last case, we further require that $t_{i}$ is of sort $s_{i}$ and $\operatorname{ar}(f)=s_{1} \times \ldots \times s_{k} \rightarrow$ $s$. We also allow subsorts: if $s_{2}$ is a subsort of $s_{1}$ we allow a term of sort $s_{2}$ whenever a term of sort $s_{1}$ is expected. We write $\operatorname{sort}(t)$ for the sort of term $t$. We define $\operatorname{root}(t)$ to be $f$ if $t=f\left(t_{1}, \ldots, t_{n}\right)$ and $t$ otherwise, i.e. if $t$ is either a name or a variable. We write 
$\operatorname{var}(t)$ and names $(t)$ for the set of variables and names occurring in $t$, respectively. A term $t$ is ground or closed if $\operatorname{var}(t)=\emptyset$.

Substitutions are written $\sigma=\left\{x_{1} \mapsto t_{1}, \ldots, x_{n} \mapsto t_{n}\right\}$ with domain $\operatorname{dom}(\sigma)=$ $\left\{x_{1}, \ldots, x_{n}\right\}$. We only consider well-sorted, cycle-free substitutions. Such a $\sigma$ is closed if all of the $t_{i}$ are closed. We let $\operatorname{var}(\sigma)=\bigcup_{i} \operatorname{var}\left(t_{i}\right)$, names $(\sigma)=\bigcup_{i} \operatorname{names}\left(t_{i}\right)$, and extend the notations $\operatorname{var}($.$) and names(.) to tuples and sets of terms and substitutions$ in the obvious way. The application of a substitution $\sigma$ to a term $t$ is written $\sigma(t)=$ $t \sigma$ and is defined in the usual way. As usual the set of positions pos $(t)$ of a term $t$ is defined inductively as $\operatorname{pos}(c)=\operatorname{pos}(a)=\operatorname{pos}(x)=\{\epsilon\}$ where $\operatorname{ar}(c)=s$ and $\operatorname{pos}\left(f\left(t_{1}, \ldots, t_{n}\right)\right)=\{\epsilon\} \cup \bigcup_{1 \leq i \leq n} i \cdot \operatorname{pos}\left(t_{i}\right)$. If $p$ is a position of $t$ then expression $\left.t\right|_{p}$ denotes the subterm of $t$ at the position $p$, i.e., $\left.t\right|_{\epsilon}=t$ and $\left.f\left(t_{1}, \ldots, t_{n}\right)\right|_{i \cdot p}=\left.t_{i}\right|_{p}$.

Symbols in $\mathcal{F}$ are intended to model cryptographic primitives, whereas names in $\mathcal{N}$ are used to model secrets, e.g., keys. The abstract semantics of symbols is described by an equational theory $E$, i.e, an equivalence relation (also written $=_{E}$ ) which is stable by application of contexts and substitutions of variables. For instance, symmetric encryption can be modeled by the classical theory $E_{\text {enc }}=\{\operatorname{dec}(\operatorname{enc}(x, y), y)=x\}$.

\subsection{Deducibility and Static Equivalence}

We use frames [3|2] to represent sequences of messages observed by an attacker. Formally, a frame is an expression $\varphi=\nu \tilde{a} .\left\{x_{1}=t_{1}, \ldots, x_{n}=t_{n}\right\}$ where $\tilde{a}$ is a set of bound (or restricted) names, and for each $i, t_{i}$ is a closed term of the same sort as $x_{i}$. For simplicity, we only consider frames $\varphi=\nu \tilde{a} .\left\{x_{1}=t_{1}, \ldots, x_{n}=t_{n}\right\}$ which restrict every name in use, i.e., $\tilde{a}=\operatorname{names}\left(t_{1}, \ldots, t_{n}\right)$. A name $a$ may still be disclosed explicitly by adding a mapping $x_{a}=a$ to the frame. Thus we assimilate such frames $\varphi$ to their underlying substitutions $\sigma=\left\{x_{1} \mapsto t_{1}, \ldots, x_{n} \mapsto t_{n}\right\}$ also denoted $\left\{x_{i} \mapsto t_{i}\right\}_{1 \leq i \leq n}$.

Definition 1 (Deducibility). A (closed) term $t$ is deducible from a frame $\varphi$ in an equational theory $E$, written $\varphi \vdash_{E} t$, iff there exists a term $M$ such that $\operatorname{var}(M) \subseteq \operatorname{dom}(\varphi)$, $\operatorname{names}(M) \cap \operatorname{names}(\varphi)=\emptyset$, and $M \varphi={ }_{E} t$.

For simplicity we only consider deducibility problems $\varphi \vdash_{E} t$ such that names $(t) \subseteq$ $\operatorname{names}(\varphi)$. For instance, let $\varphi_{1}=\left\{x_{1} \mapsto \operatorname{enc}\left(k_{1}, k_{2}\right), x_{2} \mapsto \operatorname{enc}\left(k_{4}, k_{3}\right), x_{3} \mapsto k_{3}\right\}$ : under the the theory $E_{\text {enc }}$ name $k_{4}$ is deducible from $\varphi_{1} \operatorname{since} \operatorname{dec}\left(x_{2}, x_{3}\right) \varphi_{1}={ }_{E_{\text {enc }}} k_{4}$ but neither are $k_{1}$ nor $k_{2}$. As also argued in [2] deducibility is not always sufficient to account for the knowledge of an attacker. For instance, it lacks partial information on secrets. That is why another classical notion in formal methods is static equivalence.

Definition 2 (Static equivalence). Two frames $\varphi_{1}$ and $\varphi_{2}$ are statically equivalent in a theory $E$, written $\varphi_{1} \approx_{E} \varphi_{2}$, iff $\operatorname{dom}\left(\varphi_{1}\right)=\operatorname{dom}\left(\varphi_{2}\right)$, and for all terms $M$ and $N$ such that $\operatorname{var}(M, N) \subseteq \operatorname{dom}\left(\varphi_{1}\right)$ and $\operatorname{names}(M, N) \cap \operatorname{names}\left(\varphi_{1}, \varphi_{2}\right)=\emptyset, M \varphi_{1}={ }_{E} N \varphi_{1}$ is equivalent to $M \varphi_{2}={ }_{E} N \varphi_{2}$.

For instance, let 0 and 1 be two constants (which are known by the attacker). Then $\{x \mapsto \operatorname{enc}(0, k)\} \approx_{E_{\text {enc }}}\{x \mapsto \operatorname{enc}(1, k)\}$. However $\varphi=\{x \mapsto \operatorname{enc}(0, k), y \mapsto k\}$ and $\varphi^{\prime}=\{x \mapsto \operatorname{enc}(1, k), y \mapsto k\}$ are not statically equivalent for $E_{\text {enc }}$ : let $M=\operatorname{dec}(x, y)$ and $N=0 . M$ and $N$ use only variables defined by $\varphi$ and $\varphi^{\prime}$ and do not use any names. Moreover $M \varphi=E_{\text {enc }} N \varphi$ but $M \varphi \neq_{E_{\text {enc }}} N \varphi$. The test $M \stackrel{?}{=} N$ distinguishes $\varphi$ from $\varphi^{\prime}$. 


\subsection{Concrete Semantics}

We now give terms and frames a concrete semantics, parameterized by an implementation of the primitives. Provided a set of sorts $\mathcal{S}$ and a set of symbols $\mathcal{F}$ as above, a $(\mathcal{S}, \mathcal{F})$-computational algebra $A$ consists of

- a non-empty set of bit-strings $\llbracket s \rrbracket_{A} \subseteq\{0,1\}^{*}$ for each sort $s \in \mathcal{S}$; moreover, if $s_{2}$ is a subsort of $s_{1}$ we require that $\llbracket s_{2} \rrbracket_{A} \subseteq \llbracket s_{1} \rrbracket_{A}$;

- a computable function $\llbracket f \rrbracket_{A}: \llbracket s_{1} \rrbracket_{A} \times \ldots \times \llbracket s_{k} \rrbracket_{A} \rightarrow \llbracket s \rrbracket_{A}$ for each $f \in \mathcal{F}$ with $\operatorname{ar}(f)=s_{1} \times \ldots \times s_{k} \rightarrow s$;

- an effective procedure to draw random elements from $\llbracket s \rrbracket_{A}$, denoted $x \stackrel{R}{\longleftarrow} \llbracket s \rrbracket_{A}$.

Assume a fixed $(\mathcal{S}, \mathcal{F})$-computational algebra $A$. We associate to each frame $\varphi=$ $\left\{x_{1} \mapsto t_{1}, \ldots, x_{n} \mapsto t_{n}\right\}$ a distribution $\psi=\llbracket \varphi \rrbracket_{A}$, of which the drawings $\widehat{\psi} \stackrel{R}{\longleftarrow} \psi$ are computed as follows:

1. for each name $a$ of sort $s$ appearing in $t_{1}, \ldots, t_{n}$, draw a value $\widehat{a} \stackrel{R}{\longleftarrow} \llbracket s \rrbracket_{A}$;

2. for each $x_{i}(1 \leq i \leq n)$ of sort $s_{i}$, compute $\widehat{t_{i}} \in \llbracket s_{i} \rrbracket_{A}$ recursively on the structure of terms: $f\left(\widehat{t_{1}^{\prime}, \ldots,} t_{m}^{\prime}\right)=\llbracket f \rrbracket_{A}\left(\widehat{t_{1}^{\prime}}, \ldots, \widehat{t_{m}^{\prime}}\right)$;

3. return the value $\widehat{\psi}=\left\{x_{1} \mapsto \widehat{t_{1}}, \ldots, x_{n} \mapsto \widehat{t_{n}}\right\}$.

Such values $\phi=\left\{x_{1}=e_{1}, \ldots, x_{n}=e_{n}\right\}$ with $e_{i} \in \llbracket s_{i} \rrbracket_{A}$ are called concrete frames. We extend the notation $\llbracket . \rrbracket_{A}$ to (tuples of) closed terms in the obvious way. We also generalize the notation to terms with variables, by specifying the concrete values for all of them: $\llbracket \cdot \rrbracket_{A,\left\{x_{1}=e_{1}, \ldots, x_{n}=e_{n}\right\}}$.

In the rest of the paper we focus on asymptotic notions of cryptographic security and consider families of computational algebra $\left(A_{\eta}\right)$ indexed by a complexity parameter $\eta \geq 0$. (This parameter $\eta$ might be thought of as the size of keys and other secret values.) The concrete semantics of a frame $\varphi$ is a family of distributions over concrete frames $\left(\llbracket \varphi \rrbracket_{A_{\eta}}\right)$. We only consider families of computational algebras $\left(A_{\eta}\right)$ such that each required operation on algebras is feasible by a (uniform, probabilistic) polynomialtime algorithm in the complexity parameter $\eta$. This ensures that the concrete semantics of terms and frames is efficiently computable (in the same sense).

Families of distributions (ensembles) over concrete frames benefit from the usual notion of cryptographic indistinguishability. Intuitively, two families of distributions $\left(\psi_{\eta}\right)$ and $\left(\psi_{\eta}^{\prime}\right)$ are indistinguishable, written $\left(\psi_{\eta}\right) \approx\left(\psi_{\eta}^{\prime}\right)$, if and only if no probabilistic polynomial-time adversary $\mathcal{A}$ can guess whether he is given a sample from $\psi_{\eta}$ or $\psi_{\eta}^{\prime}$ with a probability significantly greater than $\frac{1}{2}$. Formally, we ask the advantage of $\mathcal{A}$,

$$
\operatorname{Adv}_{\mathcal{A}}^{\mathrm{IND}}\left(\psi_{\eta}, \psi_{\eta}^{\prime}\right)=\left|\mathbb{P}\left[\widehat{\psi} \stackrel{R}{\longleftarrow} \psi_{\eta}: \mathcal{A}(\widehat{\psi})=1\right]-\mathbb{P}\left[\widehat{\psi} \stackrel{R}{\longleftarrow} \psi_{\eta}^{\prime}: \mathcal{A}(\widehat{\psi})=1\right]\right|
$$

to be a negligible function of $\eta$, that is, to remain eventually smaller than any $\eta^{-n}$ ( $n>$ 0 ) for sufficiently large $\eta$. By convention, the adversaries are given access implicitly to as many fresh random coins as needed, as well as the complexity parameter $\eta$. 


\section{Adaptive Soundness}

In this section, we recall the original notion of soundness for static equivalence which considers a passive adversary [9] and then extend it to an adaptive adversary. We show relations between the classical soundness and our new adaptive soundness and also provide a combination result which allows us, under some hypotheses, to prove adaptive soundness of computational algebras $\left(A_{\eta}\right)$ from adaptive soundness of parts of $\left(A_{\eta}\right)$.

\subsection{Soundness Definitions}

Definition 3 ( $\approx_{E}$-soundness). Let $E$ be an equational theory. A family of computational algebras $\left(A_{\eta}\right)$ is $\approx_{E}$-sound iff for every frames $\varphi_{1}, \varphi_{2}$ with the same domain, $\varphi_{1} \approx_{E} \varphi_{2}$ implies that $\left(\llbracket \varphi_{1} \rrbracket_{A_{\eta}}\right) \approx\left(\llbracket \varphi_{2} \rrbracket_{A_{\eta}}\right)$,

Similarly, Baudet et al. [9] define soundness for $=_{E}$ and $\vdash_{E}$. We here concentrate on soundness of static equivalence. As shown in [9], for many theories soundness of static equivalence implies all of the other notions. Baudet et al. also introduce a strong notion of soundness that holds without restriction on the computational power of adversaries.

Definition 4 (Unconditional $\approx_{E}$-Soundness). Let $E$ be an equational theory. A family of computational algebras $\left(A_{\eta}\right)$ is unconditionally $\approx_{E}$-sound iff for every frames $\varphi_{1}, \varphi_{2}$ with the same domain, $\varphi_{1} \approx_{E} \varphi_{2}$ implies $\left(\llbracket \varphi_{1} \rrbracket_{A_{\eta}}\right)=\left(\llbracket \varphi_{2} \rrbracket_{A_{\eta}}\right)$.

Unconditional soundness stipulates that for any pairs of equivalent frames, the related distributions are equal. Hence even an adversary which is not polynomially bounded cannot distinguish these two distributions.

\subsection{Adaptive Security}

We extend soundness of static equivalence to the adaptive setting from [24] where the adversary observes the computational value of a sequence of adaptively chosen terms.

The adaptive setting is formalized through the following cryptographic game. Let $\left(A_{\eta}\right)$ be a family of computational algebras and $\mathcal{A}$ be an adversary. $\mathcal{A}$ has access to a left-right evaluation oracle $\mathcal{O}_{L R}$ which given a pair of symbolic terms $\left(t_{0}, t_{1}\right)$ outputs either the implementation of $t_{0}$ or of $t_{1}$. This oracle depends on a selection bit $b$ and uses a local store to record values generated for the different names (these values are used when processing further queries). With a slight abuse of notation, we omit this store and write: $\mathcal{O}_{L R, A_{\eta}}^{b}\left(t_{0}, t_{1}\right)=\llbracket t_{b} \rrbracket_{A_{\eta}}$. Adversary $\mathcal{A}$ plays an indistinguishability game with the objective of finding the value of $b$. Formally the advantage of $\mathcal{A}$ is defined by:

$$
\operatorname{Adv}_{\mathcal{A}, A_{\eta}}^{\mathrm{ADPT}}(\eta)=\left|\mathbb{P}\left[\mathcal{A}^{\mathcal{O}_{L R, A_{\eta}}^{1}}=1\right]-\mathbb{P}\left[\mathcal{A}^{\mathcal{O}_{L R, A_{\eta}}^{0}}=1\right]\right|
$$

Without further restrictions on the queries of the adversary, having a non-negligible advantage is easy in most cases. For example the adversary could submit a pair $(0,1)$ to his oracle. We therefore require the adversary to be legal.

Definition 5 (Adaptive soundness). An adversary $\mathcal{A}$ is legal if for any sequence of queries $\left(t_{0}^{i}, t_{1}^{i}\right)_{1 \leq i \leq n}$ made by $\mathcal{A}$ to its left-right oracle, queries are statically equivalent: $\left\{x_{1} \mapsto t_{0}^{1}, \ldots, x_{n} \mapsto t_{0}^{n}\right\} \approx_{E}\left\{x_{1} \mapsto t_{1}^{1}, \ldots, x_{n} \mapsto t_{1}^{n}\right\}$. A family of computational algebras $\left(A_{\eta}\right)$ is 
- $\approx_{E}$-ad-sound iff $\operatorname{Adv}_{\mathcal{A}, A_{\eta}}^{\mathrm{ADPT}}(\eta)$ is negligible for any probabilistic polynomial-time legal adversary $\mathcal{A}$.

- unconditionally $\approx_{E}$-ad-sound iff $\operatorname{Adv}_{\mathcal{A}, A_{\eta}}^{\mathrm{ADPT}}(\eta)$ is 0 for any legal adversary $\mathcal{A}$.

Adaptive soundness implies the original soundness notion for static equivalence.

Interestingly, for unconditional soundness, the adaptive and non-adaptive case coincide.

Proposition 2. Let $\left(A_{\eta}\right)$ be a family of computational algebras. $A_{\eta}$ is unconditionally $\approx_{E}$-ad-sound iff $A_{\eta}$ is unconditionally $\approx_{E^{-} \text {-sound } .}$

\subsection{Combination Result}

Our objective here is to provide a combination result of the form: let $\Sigma_{1}$ and $\Sigma_{2}$ be two signatures that do not share any symbol. If $A_{\eta}^{1}$ is $\approx_{E_{1}}$-ad-sound and $A_{\eta}^{2}$ is $\approx_{E_{2}}$ ad-sound, then the combination of $A_{\eta}^{1}$ and $A_{\eta}^{2}$ denoted $A_{\eta}^{1} \times A_{\eta}^{2}$ is $\approx_{E_{1} \cup E_{2}}$-ad-sound. However this is false in general. Therefore, we provide restrictions under which combination is possible: we consider disjoint signatures as well as layered signatures.

Definition 6 (Disjoint signatures). Let $\Sigma_{1}=\left(\mathcal{S}_{1}, \mathcal{F}_{1}\right)$ and $\Sigma_{2}=\left(\mathcal{S}_{2}, \mathcal{F}_{2}\right)$ be two signatures. We say that $\Sigma_{1}$ and $\Sigma_{2}$ are disjoint iff $\mathcal{F}_{1} \cap \mathcal{F}_{2}=\emptyset$ and $\mathcal{S}_{1} \cap \mathcal{S}_{2}=\emptyset$.

We denote by $\Sigma_{1} \cup \Sigma_{2}=\left(\mathcal{S}_{1} \cup \mathcal{S}_{2}, \mathcal{F}_{1} \cup \mathcal{F}_{2}\right)$ the union of the signature $\Sigma_{1}$ with $\Sigma_{2}$.

Definition 7 (Signature combination, layered signatures). Let $\Sigma_{1}=\left(\mathcal{S}_{1}, \mathcal{F}_{1}\right)$ and $\Sigma_{2}=\left(\mathcal{S}_{2}, \mathcal{F}_{2}\right)$ be two disjoint signatures. A subsort relation $\mathrm{S}$ is a signature combination for $\Sigma_{1}$ and $\Sigma_{2}$ if $\mathrm{S} \subseteq \mathcal{S}_{2} \times \mathcal{S}_{1}$. Then $\Sigma=\Sigma_{1} \cup \Sigma_{2}$ is a $\left(\Sigma_{1}, \Sigma_{2}\right)_{\text {s-layered }}$ signature.

Intuitively, if a signature is layered then a constructor of $\mathcal{F}_{1}$ never occurs under a constructor of $\mathcal{F}_{2}$ and $S$ defines which sorts of $\Sigma_{2}$ can be used as subsort of $\Sigma_{1}$. Given a $\left(\Sigma_{1}, \Sigma_{2}\right)_{\mathrm{S}}$-layered signature $\Sigma$ and a term $t$ over $\Sigma$ we define the set of $\Sigma_{1}$ positions of $t, \operatorname{pos}_{\Sigma_{1}}(t)=\left\{p \mid p \in \operatorname{pos}(t), \operatorname{sort}\left(\left.t\right|_{p}\right) \in \mathcal{S}_{1}\right\}$ and the set of $\Sigma_{2}$ minimal positions of $t, \operatorname{pos}_{\Sigma_{2}}^{\star}(t)=\left\{p \mid p \in \operatorname{pos}(t), \operatorname{sort}\left(\left.t\right|_{p}\right) \in \mathcal{S}_{2}, p=p^{\prime} \cdot i \Rightarrow \operatorname{sort}\left(\left.t\right|_{p^{\prime}}\right) \notin \mathcal{S}_{2}\right\}$.

As an example consider a theory with symmetric encryption and a pseudo-random generator. Signature $\Sigma_{1}$ contains a sort Data and two symbols enc and dec, both of arity Data $\times$ Data $\rightarrow$ Data. Signature $\Sigma_{2}$ contains one sort Rand and a symbol prg (a pseudo-random generator) of arity Rand $\rightarrow$ Rand. The signature combination $S$ contains a single element (Rand, Data): elements of sort Rand can be used as keys or as plaintext. $\Sigma_{1}$ and $\Sigma_{2}$ are disjoint, and $\Sigma=\Sigma_{1} \cup \Sigma_{2}$ is $\left(\Sigma_{1}, \Sigma_{2}\right)_{\text {s-layered. Given }}$ the term $t=\operatorname{enc}\left(\operatorname{enc}(\operatorname{prg}(r), k), \operatorname{prg}\left(\operatorname{prg}\left(r^{\prime}\right)\right)\right)$ where $k \in$ Data and $r, r^{\prime} \in$ Rand, $t$ is indeed a valid term of $\Sigma$. However, the term $t^{\prime}=\operatorname{prg}(\operatorname{enc}(r, k))$ is not a term of $\Sigma$ as it is not well sorted. We have that $\operatorname{pos}_{\Sigma_{1}}(t)=\{\epsilon, 1,12\}$ and $\operatorname{pos}_{\Sigma_{2}}^{\star}=\{11,2\}$.

Definition 8 (Hybrid functions). Let $\Sigma_{1}, \Sigma_{2}$ be two disjoint signatures and S a signature combination such that $\Sigma=\Sigma_{1} \cup \Sigma_{2}$ is $\left(\Sigma_{1}, \Sigma_{2}\right)_{\mathrm{S}}$-layered. Let $E_{1}$, $E_{2}$ be equational theories over $\Sigma_{1}$ and $\Sigma_{2}$ respectively. $A\left(E_{1}, E_{2}\right)$-hybrid function for a set $F$ of pairs of frames is a function $\sigma$ from lists of terms over $\Sigma$ to terms over $\Sigma$ such that: 
- for any frame $\varphi$ occurring in $F, \varphi \approx_{E_{1}} \sigma(\varphi)$ where we naturally extended $\sigma$ over frames by $\sigma\left(\left\{x_{1} \mapsto t_{1}, \ldots, x_{n} \mapsto t_{n}\right\}\right)=\left\{x_{1} \mapsto \sigma\left(\left[t_{1}\right]\right), \ldots, x_{n} \mapsto\right.$ $\left.\sigma\left(\left[t_{1} \ldots t_{n}\right]\right)\right\}$;

- for any $\left(\varphi, \varphi^{\prime}\right) \in F$, if $\varphi \approx_{E_{1} \cup E_{2}} \varphi^{\prime}$ then let $\varphi=\left\{x_{1} \mapsto t_{1}, \ldots, x_{n} \mapsto t_{n}\right\}$ and $\varphi^{\prime}=\left\{x_{1} \mapsto u_{1}, \ldots, x_{n} \mapsto u_{n}\right\}$. We have that for all $i$ in $[1, n]$,

- $\operatorname{pos}_{\Sigma_{1}}\left(\sigma\left(\left[t_{1} \ldots t_{i}\right]\right)\right)=\operatorname{pos}_{\Sigma_{1}}\left(\sigma\left(\left[u_{1} \ldots u_{i}\right]\right)\right)=P$ and for any $p \in P$

$$
\operatorname{root}\left(\left.\sigma\left(\left[t_{1} \ldots t_{i}\right]\right)\right|_{p}\right)=\operatorname{root}\left(\left.\sigma\left(\left[u_{1} \ldots u_{i}\right]\right)\right|_{p}\right)
$$

- $\operatorname{pos}_{\Sigma_{2}}^{\star}\left(\sigma\left(\left[t_{1} \ldots t_{i}\right]\right)\right)=\operatorname{pos}_{\Sigma_{2}}^{\star}\left(\sigma\left(\left[u_{1} \ldots u_{i}\right]\right)\right)=Q$ and we have that

$$
\left\{\left.x_{q} \mapsto \sigma\left(\left[t_{1} \ldots t_{i}\right]\right)\right|_{q}\right\}_{q \in Q} \approx_{E_{2}}\left\{\left.x_{q} \mapsto \sigma\left(\left[u_{1} \ldots u_{i}\right]\right)\right|_{q}\right\}_{q \in Q}
$$

Moreover $\sigma$ has to be computable in polynomial time (in its input).

Adaptive soundness may not hold on all frames, but only on a subset of well-formed frames, e.g., when considering encryption one typically discards all frames that contain

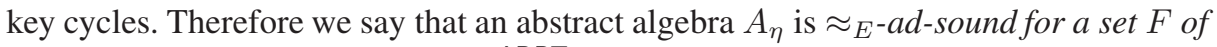
pair offrames if the advantage $\operatorname{Adv}_{\mathcal{A}, A_{\eta}}^{\mathrm{ADPT}}(\eta)$ of any polynomial-time legal adversary $\mathcal{A}$, whose sequence of queries $\left(t_{0}^{i}, t_{1}^{i}\right)_{i}$ verifies that the pair $\left(\left\{x_{i} \mapsto t_{0}^{i}\right\}_{i},\left\{x_{i} \mapsto t_{1}^{i}\right\}_{i}\right)$ is in $F$, is negligible. We typically show soundness for the set of all pairs of "well-formed" frames (the notion of well-formed frames depends on the particular equational theory).

Proposition 3 (Combination). Let $\Sigma_{1}$ and $\Sigma_{2}$ be two disjoint signatures and $\mathrm{S}$ be a signature combination for $\Sigma_{1}$ and $\Sigma_{2}$. Let $E_{1}$ and $E_{2}$ be equational theories over $\Sigma_{1}$ and $\Sigma_{2}$ respectively. We consider a family of computational algebras $\left(A_{\eta}^{1}\right)$ for $\Sigma_{1}$ and anotherfamily $\left(A_{\eta}^{2}\right)$ for $\Sigma_{2}$ respecting S, i.e. $\left(s_{2}, s_{1}\right) \in \mathrm{S}$ implies that $\llbracket s_{2} \rrbracket A_{\eta}^{2} \subseteq \llbracket s_{1} \rrbracket_{A_{\eta}^{1}}$.

Let $F$ be a set of pair of frames over $\Sigma_{1} \cup \Sigma_{2}$ and $\sigma$ be a $\left(E_{1}, E_{2}\right)$-hybrid function for $F$. If $A_{\eta}^{1} \times A_{\eta}^{2}$ is $\approx_{E_{1}}$-ad-sound for $G=\{(\varphi, \sigma(\varphi)) \mid \varphi$ occurs in $F\}$ and $A_{\eta}^{2}$ is $\approx_{E_{2}}$-ad-sound for frames on $\Sigma_{2}$, then $A_{\eta}^{1} \times A_{\eta}^{2}$ is $\approx_{E_{1} \cup E_{2}}$-ad-sound for $F$.

The idea of the proof is that if an adversary $\mathcal{A}$ against $E_{1} \cup E_{2}$-ad-soundness queries his oracle with a pair of frames $\left(\varphi, \varphi^{\prime}\right)$ in $F$ then it is possible to build an adversary $\mathcal{B}_{1}$ against $E_{1}$-ad-soundness who submits $(\varphi, \sigma(\varphi))$ to his oracle, an adversary $\mathcal{B}_{2}$ against $E_{2}$-ad-soundness who submits $\left(\sigma(\varphi), \sigma\left(\varphi^{\prime}\right)\right)$ and an adversary $\mathcal{B}_{3}$ against $E_{1}$-ad-soundness who submits $\left(\sigma\left(\varphi^{\prime}\right), \varphi^{\prime}\right)$ such that the advantages of $\mathcal{A}, \mathcal{B}_{1}, \mathcal{B}_{2}$ and $\mathcal{B}_{3}$ are related. This combination result will be useful in Section 4 when combining encryption with modular exponentiation.

\section{Adaptively Sound Theories}

We now present adaptive soundness results for several equational theories. We consider probabilistic symmetric encryption and try to be as close as possible to the models from [4] and from [24]. We assume that the implementation of the symmetric encryption scheme is semantically secure [21] and use a relevant formal theory. 
Symbolic model. Our symbolic model consists of the set of sorts $\mathcal{S}=\{$ Data $\}$, an infinite number of names for sort Data called keys and the function symbols:
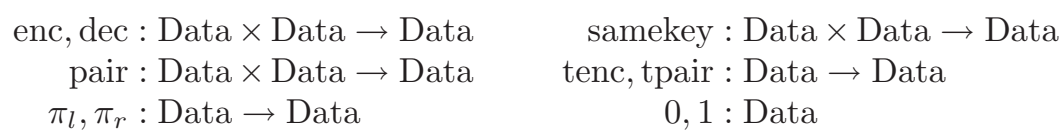

We consider the equational theory $E_{\text {sym }}$ generated by:

$$
\begin{aligned}
\operatorname{dec}(\operatorname{enc}(x, y), y) & =x & \pi_{l}(\operatorname{pair}(x, y)) & =x \\
\pi_{r}(\operatorname{pair}(x, y)) & =y & \operatorname{samekey}(\operatorname{enc}(x, y), \operatorname{enc}(z, y)) & =1 \\
\operatorname{tenc}(\operatorname{enc}(x, y)) & =1 & \operatorname{tpair}(\operatorname{pair}(x, y)) & =1
\end{aligned}
$$

Intuitively, the function symbols tenc, tpair are type testers. The meaning of the remaining symbols should be clear. As usual enc $(t, k)$ is also written $\{t\}_{k}$ and pair $\left(t, t^{\prime}\right)$ is also written $\left(t, t^{\prime}\right)$. A name $k$ is used at a key position in a term $t$ if there exists a sub-term enc $\left(t^{\prime}, k\right)$ of $t$. Else $k$ is used at a plaintext position.

Well-formed frames and adversaries. The importance of key cycles was already described in [4]. In general IND-CPA is not sufficient to prove any soundness result in the presence of key cycles. Thus, as in numerous previous work, we forbid the formal terms to contain such cycles. Let $\prec$ be a total order among keys. A frame $\varphi$ is acyclic for $\prec$ if for any subterm $\{t\}_{k}$ of $\varphi$, if $k^{\prime}$ occurs in $t$ then $k^{\prime} \prec k$. (Another possibility to handle key cycles is to consider stronger computational requirements like Key Dependent Message - KDM - security as done in [5].) Moreover as noted in [24], selective decommitment [20] can be a problem. The classical solution to this problem is to require keys to be sent before being used to encrypt a message or they must never appear as a plaintext. A frame $\varphi=\left\{x_{1} \mapsto t_{1}, \ldots, x_{n} \mapsto t_{n}\right\}$ is well-formed for $\prec$ if

$-\varphi$ is acyclic for $\prec$;

- the terms $t_{i}$ only use symbols enc, pair, 0 and 1 , and only names are used at key positions;

- if $k$ is used as plaintext in $t_{i}$, then $k$ cannot be used at a key position in $t_{j}$ for $j<i$.

An adversary is well-formed for $\prec$ if the sequence of queries $\left(t_{0}^{i}, t_{1}^{i}\right)_{1 \leq i \leq n}$ that he makes to his oracle yields two well-formed frames $\left\{x_{1} \mapsto t_{0}^{1}, \ldots, x_{n} \mapsto t_{0}^{n}\right\}$ and $\left\{x_{1} \mapsto t_{1}^{1}, \ldots, x_{n} \mapsto t_{1}^{n}\right\}$ for $\prec$.

Concrete model. A symmetric encryption scheme $\mathcal{S E}$ is defined by three algorithms $\mathcal{K} \mathcal{G}, \mathcal{E}$ and $\mathcal{D}$. The key generation algorithm takes as input the security parameter $\eta$ and outputs a key $k$. The encryption algorithm $\mathcal{E}$ is randomized. It takes as input a bit-string $s$, a key $k$ and returns the encryption of $s$ using $k$. The decryption algorithm $\mathcal{D}$ takes as input a bit-string $c$ (a ciphertext), a key $k$ and outputs the corresponding plaintext. Given $k \leftarrow \mathcal{K G}(\eta)$, for any bit-string $s$, if $c \leftarrow \mathcal{E}(k, s)$ then $\mathcal{D}(c)=s$.

The family of computational algebras $\left(A_{\eta}\right)$ giving the concrete semantics depends on a symmetric encryption scheme $\mathcal{S E}=(\mathcal{K} \mathcal{G}, \mathcal{E}, \mathcal{D})$. The concrete domain $\llbracket$ Data $\rrbracket_{A_{\eta}}$ contains all the possible bit-strings and is equipped with the distribution induced by $\mathcal{K G}$. Interpretation for constants 0 and 1 are respectively bit-strings $0^{\eta}$ and $1^{\eta}$. The enc and 
dec function are respectively interpreted using algorithm $\mathcal{E}$ and $\mathcal{D}$. We assume the existence in the concrete model of a concatenation operation which is used to interpret the pair symbol. The corresponding left and right projections implement $\pi_{l}$ and $\pi_{r}$. Finally, as we are only interested in well-formed frames, we do not provide any computational interpretation for tenc, tpair and samekey.

Semantic security. In this paper we use schemes that satisfy length-concealing semantic security. The definition that we recall below uses a left-right encryption oracle $L R_{\mathcal{S E}}^{b}$. This oracle first generates a key $k$ using $\mathcal{K G}$. Then it answers queries of the form $\left(b s_{0}, b s_{1}\right)$, where $b s_{0}$ and $b s_{1}$ are bit-strings. The oracle returns ciphertext $\mathcal{E}\left(b s_{b}, k\right)$. The goal of the adversary $\mathcal{A}$ is to guess the value of bit $b$. His advantage is defined as:

$$
\operatorname{Adv}_{\mathcal{S E}, \mathcal{A}}^{c p a}(\eta)=\mid \mathbb{P}\left[\mathcal{A}^{L R_{\mathcal{S E}}^{1}}=1\right]-\mathbb{P}\left[\mathcal{A}^{\left.L R_{\mathcal{S E}}^{0}=1\right] \mid}\right.
$$

Encryption scheme $\mathcal{S E}$ is IND-CPA secure if the advantage of any adversary $\mathcal{A}$ is negligible in $\eta$. The difference with standard semantic security is that we require the scheme to hide the length of the plaintext (and therefore we do not restrict $b s_{0}$ and $b s_{1}$ to have equal length). By abuse of notation we call the resulting scheme also IND-CPA secure.

Proposition 4. Let $\prec$ be a total order among keys. In the remainder of this proposition we only consider well-formed adversaries for $\prec$. Let $\left(A_{\eta}\right)$ be a family of computational algebras based on a symmetric encryption scheme $\mathcal{S E} .\left(A_{\eta}\right)$ is $\approx_{E_{\mathrm{sym}}}$-ad-sound if $\mathcal{S E}$ is IND-CPA but the converse is false.

\subsection{Exclusive OR}

We study the adaptive soundness problem for the usual theory and implementation of the Exclusive Or (XOR) in the same model as given in [9]. The symbolic model $\Sigma_{\oplus}$ consists of a single sort Data $\oplus$, an infinite number of names, the infix symbol $\oplus$ : Data $_{\oplus} \times$ Data $_{\oplus} \rightarrow$ Data $_{\oplus}$ and two constants $0_{\oplus}, 1_{\oplus}$ : Data $\oplus$. Terms are equipped with the equational theory $E_{\oplus}$ generated by:

$$
(x \oplus y) \oplus z=x \oplus(y \oplus z) \quad x \oplus y=y \oplus x \quad x \oplus x=0_{\oplus} \quad x \oplus 0_{\oplus}=x
$$

As an implementation, we define the computational algebras $A_{\eta}$ : the concrete domain $\llbracket$ Data $_{\oplus} \rrbracket_{A_{\eta}}$ is $\{0,1\}^{\eta}$ equipped with the uniform distribution; $\oplus$ is interpreted by the usual XOR function over $\{0,1\}^{\eta}, \llbracket 0_{\oplus} \rrbracket_{A_{\eta}}=0^{\eta}, \llbracket 1_{\oplus} \rrbracket_{A_{\eta}}=1^{\eta}$. This implementation of $\mathrm{XOR}$ enjoys unconditional adaptive soundness with respect to $\approx_{E_{\oplus}}$.

Proposition 5. The usual implementation for $E_{\oplus}$ is unconditionally $\approx_{E_{\oplus}}$-ad-sound.

The result follows directly from unconditionally $\approx_{E_{\oplus}}$-soundness shown in [9] and Proposition 2 .

\subsection{Modular Exponentiation}

As a third application, we study soundness of modular exponentiation. The underlying cryptographic assumption is hardness of the Decisional Diffie-Hellman (DDH) problem: given $g^{x}$ and $g^{y}$, it is difficult for any feasible computation to distinguish between 
$g^{x y}$ and $g^{r}$, when $x, y$ and $r$ are selected at random. The original Diffie-Hellman protocol has been used as a building block for several key agreement protocols that are widely used in practice (e.g. SSL/TLS and Kerberos V5) as well as for group key exchange protocols such as AKE1 [12] or the Burmester-Desmedt protocol [14].

Symbolic model. The symbolic model consists of sorts $G$ (group elements) and $R$ (ring elements), an infinite number of names for $R$ (but no name for sort $G$ ) and the symbols:

$$
\begin{array}{cccc}
\exp : R \rightarrow G & \text { exponentiation } & +, \cdot: R \times R \rightarrow R & \text { add, mult } \\
*: G \times G \rightarrow G & \text { mult in } \mathbb{G} & -: R \rightarrow R & \text { inverse } \\
& 0_{R}, 1_{R}: R & \text { constants } &
\end{array}
$$

We consider the equational theory $E_{\mathrm{DH}}$ generated by:

$$
\begin{array}{ccc}
x+y=y+x & x \cdot y=y \cdot x & (x+y)+z=x+(y+z) \\
x \cdot(y+z)=x \cdot y+x \cdot z & (x \cdot y) \cdot z=x \cdot(y \cdot z) & x+(-x)=0_{R} \\
0_{R}+x=x & 1_{R} \cdot x=x & \exp (x) * \exp (y)=\exp (x+y)
\end{array}
$$

There exists a direct correspondence between terms of sort $R$ and the set of polynomials $\mathbb{Z}\left[\mathcal{N}_{R}\right]$ where $\mathcal{N}_{R}$ is the set of names of sort $R$. An integer $i$ simply corresponds to $\underbrace{1_{R}+\ldots+1_{R}}_{i \text { times }}$ if $i>0$, to $-(\underbrace{1_{R}+\ldots+1_{R}}_{i \text { times }})$ if $i<0$ and to $0_{R}$ if $i=0$. We also write $x^{n}$ for $\underbrace{x \cdot \ldots \cdot x}_{n \text { times }}$.

We put two restrictions on formal terms: products have to be power-free, i.e., $x^{n}$ is forbidden for $n>1$, and products must not contain more than $l$ elements for some fixed bound $l$, i.e. $x_{1} \cdot \ldots \cdot x_{n}$ is forbidden for $n>l$. Both restrictions come from the DDH assumption and seem difficult to avoid [13]. Furthermore we are only interested in frames using terms of sort $G$. Any frame containing only terms of sort $G$ can be rewritten as $\left\{x_{1} \mapsto \exp \left(p_{1}\right), \ldots, x_{n} \mapsto \exp \left(p_{n}\right)\right\}$ by orienting the last equation form left to right. For such frames there is an immediate characterization of static equivalence. Two frames are statically equivalent if they satisfy the same linear equations.

Proposition 6. We have that $\left\{x_{1} \mapsto \exp \left(p_{1}\right), \ldots, x_{n} \mapsto \exp \left(p_{n}\right)\right\} \approx_{E_{\mathrm{DH}}}\left\{x_{1} \mapsto\right.$ $\left.\exp \left(q_{1}\right), \ldots, x_{n} \mapsto \exp \left(q_{n}\right)\right\}$ iff for any sequence of integer $a_{0}, a_{1}, \ldots a_{n}$ we have $a_{0}+\sum_{i=1}^{n} a_{i} p_{i}=0 \Leftrightarrow a_{0}+\sum_{i=1}^{n} a_{i} q_{i}=0$

This characterization can be used to decide static equivalence efficiently.

Concrete model. An Instance Generator $I G$ is a polynomial-time (in $\eta$ ) algorithm that outputs a cyclic group $\mathbb{G}$ (defined by a generator $g$, an order $q$ and a polynomial-time multiplication algorithm) of prime order $q$. The family of computational algebras $\left(A_{\eta}\right)$ depends on an instance generator $I G$ which generates a cyclic group $\mathbb{G}$ of generator $g$ and of order $q$ : the concrete domain $\llbracket R \rrbracket_{A_{\eta}}$ is $\mathbb{Z}_{q}$ with the uniform distribution. Symbols + and $\cdot$ are the classical addition and multiplication over $\mathbb{Z}_{q}$, exp is interpreted as modular exponentiation of $g$. Constants $0_{R}$ and $1_{R}$ are interpreted by integers 0 and 1 of $\mathbb{Z}_{q}$. The domain $\llbracket G \rrbracket_{A_{\eta}}$ contains all bit-string representations of elements of $\mathbb{G}$.

A family of computational algebras satisfies the DDH assumption if its instance generator satisfies the assumption: for every probabilistic polynomial-time adversary $\mathcal{A}$, his 
advantage $\operatorname{Adv}_{I G, \mathcal{A}}^{\mathrm{DDH}}(\eta)=\mid \mathbb{P}\left[(g, q) \leftarrow I G(\eta): a, b \leftarrow \mathbb{Z}_{q}: \mathcal{A}\left(g^{a}, g^{b}, g^{a b}\right)=1\right]-$ $\mathbb{P}\left[(g, q) \leftarrow I G(\eta): a, b, c \leftarrow \mathbb{Z}_{q}: \mathcal{A}\left(g^{a}, g^{b}, g^{c}\right)=1\right] \mid$ is negligible in $\eta$. In the remainder, we generally suppose that for any $\eta$ there is a unique group given by $I G$. We show that the DDH assumption is necessary and sufficient to prove adaptive soundness.

Proposition 7. A family of computational algebras $\left(A_{\eta}\right)$ is $\approx_{E_{\mathrm{DH}}}$-sound iff $\left(A_{\eta}\right)$ is $\approx_{E_{\mathrm{DH}}}$-ad-sound iff $\left(A_{\eta}\right)$ satisfies the $\mathrm{DDH}$ assumption.

The proof of this result uses an adaptive variant of DDH called 3DH: it generalizes several previously used variants of DDH. The main difficulty in this proof consists in relating DDH and 3DH. Note that while adaptive soundness and (classical) soundness are not equivalent for symmetric encryption, they coincide in this case.

\subsection{Combining Encryption with Exponentiation}

We illustrate our combination result (Proposition 3) by establishing a joint soundness result for symmetric encryption and modular exponentiation.

Symbolic model. We consider an equational theory $E$ containing both $E_{\mathrm{DH}}$ and $E_{\mathrm{sym}}$. Let $\Sigma_{1}$ be the signature for symmetric encryption and $\Sigma_{2}$ be the signature for modular exponentiation, then signature $\Sigma=\Sigma_{1} \cup \Sigma_{2}$ is $\left(\Sigma_{1}, \Sigma_{2}\right)_{\text {S-layered where } S \text { contains }}$ only one element $(G$, Data).

Well-formed frames. Let $\prec$ be a total order between keys and exponentiations. A frame $\varphi$ (on $\Sigma$ ) is well-formed for $\prec$ if:

- $\varphi$ does not contain any dec, tenc, tpair, $\pi_{l}, \pi_{r}$ or $*$ symbol, only names and exponentiations are used at key position.

- For any subterm $\exp (p)$ of $\varphi$ used at a key position, $p$ is linearly independent of other polynomials $p^{\prime}$ such that $\exp \left(p^{\prime}\right)$ is a subterm of $\varphi$.

- For any subterm $\{t\}_{t^{\prime}}$ of $\varphi$, if $t^{\prime \prime}$ is a subterm of $t$ which is a name of sort Data or an exponentiation then $t^{\prime \prime} \prec t^{\prime}$.

Concrete model. The concrete model is given by the models for symmetric encryption and modular exponentiation. We need to reflect that exponentiations can be used as symmetric keys. The family of computational algebras $\left(A_{\eta}\right)$ giving the concrete semantics is parameterized by a symmetric encryption scheme $\mathcal{S E}$ and an instance generator $I G$. We require that the key generation algorithm of $\mathcal{S E}$ randomly samples an element of $I G(\eta)$. Giving an IND-CPA encryption scheme $\mathcal{S} \mathcal{E}^{\prime}$, it is possible to build another IND-CPA scheme $\mathcal{S E}$ which indeed uses such a key generation algorithm. This is achieved by using a key extractor algorithm Kex [16]. This algorithm (usually a universal hash function used with the entropy smoothing theorem) transforms group elements into valid keys for $\mathcal{S} \mathcal{E}^{\prime}$. The new encryption and decryption algorithms of $\mathcal{S} \mathcal{E}$ apply the Kex algorithm to the group element which is used as key. This produces a symmetric key which can be used with the encryption and decryption algorithms of $\mathcal{S} \mathcal{E}^{\prime}$.

The family of computational algebras $\left(A_{\eta}\right)$ implementing encryption with exponentiation is said $E E$-secure if the encryption scheme $\mathcal{S E}$ is secure against IND-CPA and 
uses a key generation algorithm as described above and $I G$ satisfies the DDH assumption. Soundness is proven by applying Proposition 3 .

Proposition 8. Let $\prec$ be a total order between keys and exponentiations. An EE-secure family of computational algebras $\left(A_{\eta}\right)$ is $\approx_{E}$-ad-sound for well-formed frames for $\prec$.

A similar result is given for symmetric encryption and XOR in the full version [22].

\section{Analysis of Dynamic Group Key Exchange}

Micciancio and Panjwani exemplified their adaptive soundness result from [24] on multicast protocols. We propose another application: dynamic group key exchange protocols (DKE) such as the AKE1 protocol [12]. To keep the symbolic security notion as simple as possible we define security for protocols using only modular exponentiation: we consider a subtheory $E$ of $E_{\mathrm{DH}}$ (Section 4.2) without,,$+- 1_{R}$ and $0_{R}$ symbols and the related equations. However our definitions and soundness results can be adapted to other equational theories (e.g. symmetric encryption joint with modular exponentiation).

\subsection{Dynamic Group Protocols}

We take a simple model for DKE in the adaptive setting. A DKE protocol is described by four operations which specify the protocol. We suppose that this specification is given by four polynomial-time algorithms $(\mathcal{S}, \mathcal{J}, \mathcal{L}, \mathcal{K})$ :

- $\mathcal{S}$ initializes a new group. The algorithm takes as an input a list of users and outputs the internal state $s_{0}$ of the protocol as well as a list of formal terms which model the messages that have been exchanged during the setup phase.

- $\mathcal{J}$ and $\mathcal{L}$ take as input the state of the protocol $s$ and a list of users $U_{1}$ to $U_{n}$ (to be respectively added to or suppressed from the group) and output the updated state of the protocol $s^{\prime}$ as well as a list of formal terms representing message exchanges.

- $\mathcal{K}$ takes as input the state of the group $s$ and outputs a formal term representing the shared key of the group.

The internal state of the protocol can be thought of as the internal state of the four algorithms that describe the protocol.

We partition the set of names of sort $R$ according to the users: $n_{i}^{j}, j \in \mathbb{N}$, are the nonces generated by user $U_{i}$. We require that the formal term output by $\mathcal{K}$ only uses nonces for users that are currently in the group.

\subsection{Security in the Symbolic Model}

In our symbolic setting, the security property is expressed as reachability in a transition system. We represent the states of this transition system as a triple $\langle L, C, T\rangle$ where

- $L$ is the list of users that are currently in the group;

- $C$ is the set of corrupted users;

$-T$ is the list of formal terms sent during the protocol execution. 
We suppose that the internal state of the protocol can be recovered from the state $\langle L, C, T\rangle$ and tend to assimilate these two notions of state. We now describe the possible transitions. For convenience, we use set notations for manipulating lists.

1. $\langle\emptyset, C, \emptyset\rangle \stackrel{\mathrm{c}(U)}{\longrightarrow}\langle\emptyset, C \cup\{U\}, \emptyset\rangle$ : corruption of user $U$.

2. $\langle\emptyset, C, \emptyset\rangle \stackrel{\mathrm{s}(\mathcal{U})}{\longrightarrow}\langle\mathcal{U}, C, T\rangle$ : setup of the group given by the list of users $\mathcal{U}$, i.e., $\langle\mathcal{U}, C, T\rangle$ is computed by $\mathcal{S}(\langle\emptyset, C, \emptyset\rangle, \mathcal{U})$.

3. $\langle L, C, T\rangle \stackrel{\mathrm{j}(\mathcal{U})}{\longrightarrow}\langle L \cup \mathcal{U}, C, T\rangle \cup T^{\prime}$ : join of users in the list $\mathcal{U}$, i.e., $\left\langle L \cup \mathcal{U}, C, T \cup T^{\prime}\right\rangle$ is computed by $\mathcal{J}(\langle L, C, T\rangle, \mathcal{U})$.

4. $\langle L, C, T\rangle \stackrel{\mathrm{I}(\mathcal{U})}{\longrightarrow}\left\langle L \backslash \mathcal{U}, C, T \cup T^{\prime}\right\rangle$ : exclusion of the users in the list $\mathcal{U}$, i.e., $\langle L \backslash$ $\left.\mathcal{U}, C, T \cup T^{\prime}\right\rangle$ is computed by $\mathcal{L}(\langle L, C, T\rangle, \mathcal{U})$.

To simplify things up, we consider a static corruption model, i.e., corruption transitions only occur at the beginning of the protocol. Then a setup transition is taken followed by leave and join transitions. A DKE protocol is secure if it is impossible for an adversary to get any bit of information on the group key when no corrupted users are in the group.

Definition 9. We define a DKE protocol to be symbolically secure if for any state $\left\langle L, C, T=\left\{t_{1}, \ldots, t_{n}\right\}\right\rangle$ reachable from $\langle\emptyset, \emptyset, \emptyset\rangle$ and such that $C \cap L=\emptyset$ we have

$\left\{x_{1} \mapsto t_{1}^{\prime}, \ldots, x_{n} \mapsto t_{n}^{\prime}, y \mapsto \mathcal{K}(\langle L, C, T\rangle)\right\} \approx_{E}\left\{x_{1} \mapsto t_{1}^{\prime}, \ldots, x_{n} \mapsto t_{n}^{\prime}, y \mapsto \exp (r)\right\}$

where $r$ is a fresh nonce, $N=\left\{n_{i}^{j} \mid U_{i} \in C\right\}$ and $t_{i}^{\prime}$ is as $t_{i}$ but nonces from $N$ have been removed, i.e. if $t=\exp \left(m_{1} \cdot \ldots \cdot m_{\ell}\right)$ then $t^{\prime}=\exp \left(m_{1}^{\prime} \cdot \ldots \cdot m_{\ell^{\prime}}^{\prime}\right)$ where $\left\{m_{1}^{\prime}, \ldots, m_{\ell^{\prime}}^{\prime}\right\}=\left\{m_{1}, \ldots, m_{\ell}\right\} \backslash N$.

\subsection{Security in the Concrete Model}

We use a simplified version of the security model from [12]: some oracles in [12] are not useful anymore in the adaptive setting. Let $(\mathcal{S}, \mathcal{J}, \mathcal{L}, \mathcal{K})$ be a DKE and $\left(A_{\eta}\right)$ a family of computational algebras. Adversary $\mathcal{A}$ interacts with the group via the following five oracles which store the current state $s$ of the group and use a challenge bit $b$.

- Setup $\left(U_{1}, \ldots, U_{n}\right)$ : initializes the group using $\mathcal{S}\left(U_{1}, \ldots, U_{n}\right)$ which produces the new state $s$ and a list of formal terms $t_{1}$ to $t_{m}$. $\mathcal{A}$ is given $\llbracket t_{i} \rrbracket_{A_{\eta}}$ for any $i$ in $[1, m]$.

- Join $\left(U_{1}, \ldots, U_{n}\right)$ : users $U_{1}$ to $U_{n}$ join the group. $\mathcal{J}\left(s, U_{1}, \ldots, U_{n}\right)$ is executed and outputs state $s$ and a list of terms $t_{1}$ to $t_{m}$. $\mathcal{A}$ is given $\llbracket t_{i} \rrbracket_{A_{\eta}}$ for any $i$ in $[1, m]$.

- Leave $\left(U_{1}, \ldots, U_{n}\right)$ : users $U_{1}$ to $U_{n}$ leave the group. $\mathcal{L}\left(s, U_{1}, \ldots, U_{n}\right)$ is executed and outputs state $s$ and a list of terms $t_{1}$ to $t_{m} . \mathcal{A}$ is given $\llbracket t_{i} \rrbracket_{A_{\eta}}$ for any $i$ in $[1, m]$.

- Corrupt $(U): \mathcal{A}$ corrupts user $U$; all nonces generated by $U$ are given to $\mathcal{A}$. As $\mathcal{A}$ works in polynomial time, a polynomial number of values is sufficient.

- Test: $\mathcal{A}$ either receives the key of the group (output by $\mathcal{K}(s)$ ) if $b=1$ or a random key if $b=0$. This oracle can only be queried once.

As we consider a static corruption model, queries to the Corrupt oracle have to be done before all further queries. Then the Setup oracle is called and after that the adversary 
interleaves queries to the Join and Leave oracles. The adversary makes a final call to the Test oracle. Let $\mathcal{O}_{b}$ denote the oracles with challenge bit $b$. The advantage of an adversary $\mathcal{A}$ is given by: $\operatorname{Adv}_{\mathcal{A}, A_{\eta}}^{(\mathcal{S}, \mathcal{J}, \mathcal{L}, \mathcal{K})}(\eta)=\mathbb{P}\left[\mathcal{A}^{\mathcal{O}_{1}}=1\right]-\mathbb{P}\left[\mathcal{A}^{\mathcal{O}_{0}}=1\right]$. A DKE is secure in the concrete model if the advantage of any adversary is negligible in $\eta$.

\subsection{Soundness Result}

Our symbolic model for DKE is computationally sound: if a DKE algorithm is secure in the symbolic model, then it is secure in the computational model, provided that static equivalence is adaptively sound (remember that we consider only modular exponentiation hence static equivalence is adaptively sound under DDH).

Proposition 9. Let $\left(A_{\eta}\right)$ be a family of computational algebras and $\Pi=(\mathcal{S}, \mathcal{J}, \mathcal{L}, \mathcal{K})$ be a DKE. If $\left(A_{\eta}\right)$ is $\approx_{E}$-ad-sound and $\Pi$ is secure in the symbolic model, then $\Pi$ is secure in the concrete model.

\section{References}

1. Abadi, M., Baudet, M., Warinschi, B.: Guessing attacks and the computational soundness of static equivalence. In: Aceto, L., Ingólfsdóttir, A. (eds.) FOSSACS 2006 and ETAPS 2006. LNCS, vol. 3921, pp. 398-412. Springer, Heidelberg (2006)

2. Abadi, M., Cortier, V.: Deciding knowledge in security protocols under equational theories. In: Díaz, J., Karhumäki, J., Lepistö, A., Sannella, D. (eds.) ICALP 2004. LNCS, vol. 3142, pp. 46-58. Springer, Heidelberg (2004)

3. Abadi, M., Fournet, C.: Mobile values, new names, and secure communications. In: Proc. 28th Annual ACM Symposium on Principles of Programming Languages (POPL'01), pp. 104-115. ACM Press, New York (2001)

4. Abadi, M., Rogaway, P.: Reconciling two views of cryptography (the computational soundness of formal encryption). In: Watanabe, O., Hagiya, M., Ito, T., van Leeuwen, J., Mosses, P.D. (eds.) TCS 2000. LNCS, vol. 1872, Springer, Heidelberg (2000)

5. Adão, P., Bana, G., Herzog, J., Scedrov, A.: Soundness of formal encryption in the presence of key-cycles. In: di Vimercati, S.d.C., Syverson, P.F., Gollmann, D. (eds.) ESORICS 2005. LNCS, vol. 3679, pp. 374-396. Springer, Heidelberg (2005)

6. Backes, M., Pfitzmann, B.: Limits of the cryptographic realization of Dolev-Yao-style XOR. In: di Vimercati, S.d.C., Syverson, P.F., Gollmann, D. (eds.) ESORICS 2005. LNCS, vol. 3679, pp. 336-354. Springer, Heidelberg (2005)

7. Backes, M., Pfitzmann, B., Waidner, M.: A composable cryptographic library with nested operations. In: Proc. 10th ACM Conference on Computer and Communications Security (CCS'03), pp. 220-230. ACM Press, New York (2003)

8. Bana, G., Mohassel, P., Stegers, T.: The computational soundness of formal indistinguishability and static equivalence. In: ASIAN'06. Proc. 11th Asian Computing Science Conference, 2006. LNCS, Springer, Heidelberg (to appear)

9. Baudet, M., Cortier, V., Kremer, S.: Computationally sound implementations of equational theories against passive adversaries. In: Caires, L., Italiano, G.F., Monteiro, L., Palamidessi, C., Yung, M. (eds.) ICALP 2005. LNCS, vol. 3580, pp. 652-663. Springer, Heidelberg (2005)

10. Blanchet, B.: Automatic proof of strong secrecy for security protocols. In: Proc. 25th IEEE Symposium on Security and Privacy (SSP'04), pp. 86-100. IEEE Computer Society Press, Los Alamitos (2004) 
11. Blanchet, B.: A computationally sound mechanized prover for security protocols. In: Proc. 27th IEEE Symposium on Security and Privacy (SSP'06), pp. 140-154. IEEE Computer Society Press, Los Alamitos (2006)

12. Bresson, E., Chevassut, O., Pointcheval, D.: Provably authenticated group Diffie-Hellman key exchange - the dynamic case. In: Boyd, C. (ed.) ASIACRYPT 2001. LNCS, vol. 2248, pp. 290-309. Springer, Heidelberg (2001)

13. Bresson, E., Lakhnech, Y., Mazaré, L., Warinschi, B.: A generalization of DDH with applications to protocol analysis and computational soundness. In: Menezes, A.J. (ed.) CRYPTO 2007. LNCS, vol. 4622, pp. 482-499. Springer, Heidelberg (2007)

14. Burmester, M., Desmedt, Y.: A secure and efficient conference key distribution system (extended abstract). In: De Santis, A. (ed.) EUROCRYPT 1994. LNCS, vol. 950, pp. 275-286. Springer, Heidelberg (1995)

15. Canetti, R., Herzog, J.: Universally composable symbolic analysis of mutual authentication and key-exchange protocols (extended abstract). In: Halevi, S., Rabin, T. (eds.) TCC 2006. LNCS, vol. 3876, pp. 380-403. Springer, Heidelberg (2006)

16. Chevassut, O., Fouque, P.-A., Gaudry, P., Pointcheval, D.: Key derivation and randomness extraction. Technical Report 2005/061, Cryptology ePrint Archive (2005)

17. Cortier, V., Delaune, S., Lafourcade, P.: A Survey of Algebraic Properties Used in Cryptographic Protocols. Journal of Computer Security, 2005 (to appear)

18. Cortier, V., Warinschi, B.: Computationally sound, automated proofs for security protocols. In: Sagiv, M. (ed.) ESOP 2005. LNCS, vol. 3444, pp. 157-171. Springer, Heidelberg (2005)

19. Datta, A., Derek, A., Mitchell, J.C., Shmatikov, V., Turuani, M.: Probabilistic Polynomialtime Semantics for a Protocol Security Logic. In: Caires, L., Italiano, G.F., Monteiro, L., Palamidessi, C., Yung, M. (eds.) ICALP 2005. LNCS, vol. 3580, pp. 16-29. Springer, Heidelberg (2005)

20. Dwork, C., Naor, M., Reingold, O., Stockmeyer, L.J.: Magic functions. J. ACM 50(6), 852921 (2003)

21. Goldwasser, S., Micali, S.: Probabilistic encryption \& how to play mental poker keeping secret all partial information. In: Proc. 14th Annual ACM Symposium on Theory of Computing (STOC'82), ACM Press, New York (1982)

22. Kremer, S., Mazaré, L.: Adaptive soundness of static equivalence. Research Report LSV07-09, Laboratoire Spécification et Vérification, ENS Cachan, France, 27 pages (February 2007)

23. Laud, P.: A composable cryptographic library with nested operations. In: Proc. 12th ACM Conference on Computer and Communications Security (CCS'05), pp. 26-35. ACM Press, New York (2005)

24. Micciancio, D., Panjwani, S.: Adaptive security of symbolic encryption. In: Kilian, J. (ed.) TCC 2005. LNCS, vol. 3378, pp. 169-187. Springer, Heidelberg (2005) 